
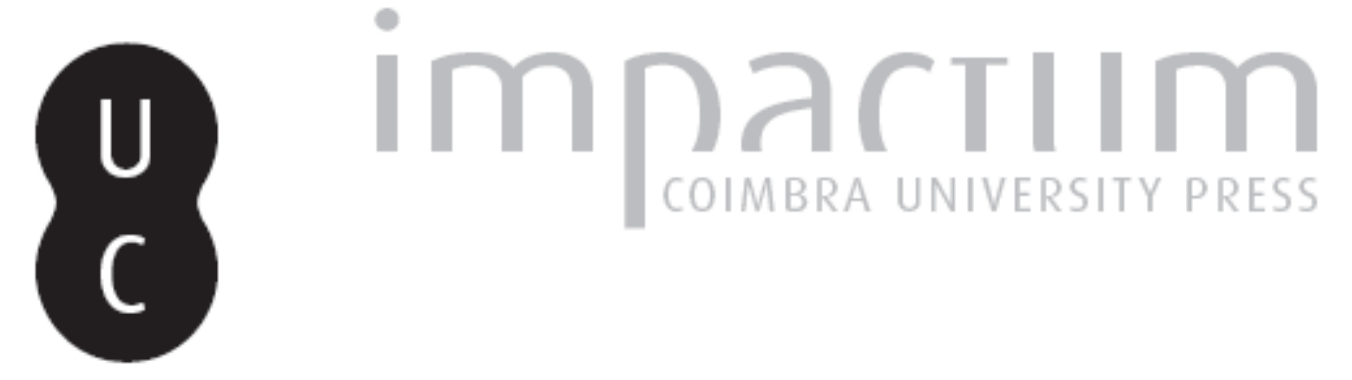

\title{
Isabel, o della 'lunga' notte
}

\section{Autor(es): Dolfi, Anna}

Publicado por: Imprensa da Universidade de Coimbra

URL persistente: URI:http://hdl.handle.net/10316.2/42434

DOI: DOI:https://doi.org/10.14195/0870-8584_8_9

Accessed : $\quad$ 26-Apr-2023 12:00:13

A navegação consulta e descarregamento dos títulos inseridos nas Bibliotecas Digitais UC Digitalis, UC Pombalina e UC Impactum, pressupõem a aceitação plena e sem reservas dos Termos e Condições de Uso destas Bibliotecas Digitais, disponíveis em https://digitalis.uc.pt/pt-pt/termos.

Conforme exposto nos referidos Termos e Condições de Uso, o descarregamento de títulos de acesso restrito requer uma licença válida de autorização devendo o utilizador aceder ao(s) documento(s) a partir de um endereço de IP da instituição detentora da supramencionada licença.

Ao utilizador é apenas permitido o descarregamento para uso pessoal, pelo que o emprego do(s) título(s) descarregado(s) para outro fim, designadamente comercial, carece de autorização do respetivo autor ou editor da obra.

Na medida em que todas as obras da UC Digitalis se encontram protegidas pelo Código do Direito de Autor e Direitos Conexos e demais legislação aplicável, toda a cópia, parcial ou total, deste documento, nos casos em que é legalmente admitida, deverá conter ou fazer-se acompanhar por este aviso.

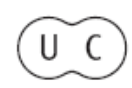




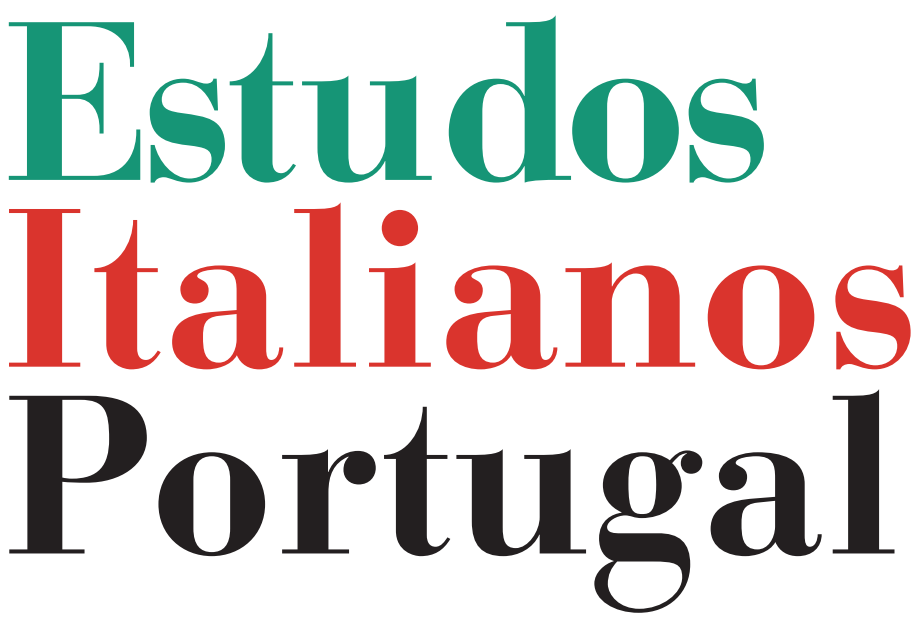

Instituto

Italiano

de Cultura

de Lisboa

Nova Série

$\mathbf{N}^{\circ} \mathbf{8}$ 


\title{
ISABEL, O DELLA 'LUNGA' NOTTE
}

\author{
ANNA DOLFI*
}

Mais la force de la Moire est inéluctable, et ni les richesses, ni Arès, ni les tours, ni les noires nefs battues des flots n'y échappent.

Sofocle, Antigone ${ }^{1}$

Io nutr[o] il dubbio che tutto, nella totalità del mondo, sia una serie intercalata di sogni e romanzi, come scatole dentro altre scatole più grandi (le une dentro le altre e queste dentro altre ancora): tutto una storia con storie, come le Mille e una Notte, a svolgersi falsamente nella notte eterna.

Fernando Pessoa, Il libro dellinquietudine $e^{2}$

\begin{abstract}
1. NonOSTANTE LA PRECISIONE dei rinvii non è facile decifrare gli esergo che Tabucchi pone in apertura dei suoi libri. Come tutti gli altri elementi del paratesto ai quali il nostro autore ricorre, svolgono una funzione tutt'altro che secondaria, visto che è loro demandato di inscrivere ogni singola storia in un
\end{abstract}

* Insegna Letteratura italiana moderna e contemporanea all'Università di Firenze. Studiosa di Leopardi e di narrativa e poesia tra fine Ottocento e Terza generazione, ha dedicato all'opera di Tabucchi un commento (Notturno indiano, 1996), due libri (Tabucchi, la specularità il rimorso, 2006; Gli oggetti e il tempo della saudade. Le storie inafferrabili di Antonio Tabucchi, 2010) e gli atti di un convegno (I 'notturni' di Antonio Tabucchi, 2008). Ha curato l'ultima raccolta di saggi dell'autore (Di tutto resta un poco. Letteratura e cinema, 2013).

${ }^{1}$ La citazione è tratta dalla traduzione di Leconte de Lisle all'Antigone di Sofocle.

${ }^{2}$ Il riferimento è al passo 133 (200) dell'edizione tabucchiana dell'immaginaria autobiografia/romanzo del grande scrittore portoghese (Fernando Pessoa, Il libro dell'inquietudine di Bernardo Soares, prefazione di Antonio Tabucchi, a cura di Maria José de Lancastre, trad. Maria José de Lancastre, Antonio Tabucchi, Milano, Feltrinelli, 2011, p. 154). 
percorso che non ha solo a che fare con l'intertestualità interna ed esterna, ma con il significato globale, si potrebbe dire 'filosofico', di quel che seguirà poco dopo. In forma interrogativa o dubitativa tracciano spesso le tappe di una riflessione che sfiora la natura del sogno o della veglia, i confini della libertà, la presenza del male, il passare del tempo. La loro intenzionalità, rafforzata talvolta dalla libertà della traduzione ${ }^{3}$, potrebbe perfino spingere a pensare che il testo inscritto nella loro 'proposizione' possa leggersi anche come una sorta di 'dimostrazione' spinoziana. Ma a partire (dato lo statuto interrogativo, problematico, costitutivo delle modalità riflessive dell'autore $^{4}$ ) da una verità solo parzialmente assiomatica anche là dove la sentenza abbia l'apparenza dell'incontestabilità.

È quanto accade con l'esergo sul quale si apre Per Isabel. Un mandala, il romanzo inedito' elaborato tra il '92 e il '96 (e dettato in quell'ultimo anno) proposto adesso dall'editore Feltrinelli. "Chissà, forse i morti hanno altro costume", con la sua sottolineatura doppiamente dubitativa ("Chissà", "forse"), d'emblée inscrive la storia che ci apprestiamo a leggere (per eccellenza fluida, mutevole, bruciata com'è dai "bar-

${ }^{3}$ È quanto succede (per limitarsi a un solo esempio) con l'esergo preposto a $\mathrm{Il}$ tempo invecchia in fretta (Milano, Feltrinelli, 2009) o con le citazioni che si trovano all'interno dei testi. In quest'ultimo campo esemplare la libera traduzione (di seconda mano, con passaggio da un testo francese) di un passo dell'Uomo senza qualità che figura in un pezzo (Per un mondo più umano) del libro postumo di saggi di Antonio Tabucchi (Di tutto resta un poco. Letteratura e cinema, a cura di Anna Dolfi, Milano, Feltrinelli, 2013, su cui Anna Dolfi, "Orizzonti. Una mappa di navigazione" [Tabucchi], Narrazioni, 3, 2013, pp. 55-64).

${ }^{4}$ Che in altro contesto mi è capitato di chiamare "andar filosofico" (cfr. "Premessa”, Il racconto e il romanzo filosofico nella modernità, a cura di Anna Dolfi, Firenze, Firenze University Press, 2013).

${ }^{5}$ Intenzionalmente inedito (nonostante la confezione compiuta: compresi l'esergo, la nota esplicativa, la quarta), e che l'autore soltanto negli ultimissimi tempi aveva pensato di recuperare, anche se non ci è dato sapere quanti e quali sarebbero stati gli interventi correttori prima di un'eventuale destinazione alla stampa (insomma è il caso di sottolineare che il libro proviene da una versione dattiloscritta per oltre quindici anni priva di revisione autoriale). 
bagli" del tempo, del passato) nel genere del requiem tanto caro a Tabucchi. Inserendola anche, tramite l'elemento della pietas politica e umana suggerita dal gesto di Antigone, nel dittico (costituito dai limitrofi Sostiene Pereira [1994], La testa perduta di Damasceno Monteiro [1997]) che assegna ai protagonisti il compito di portare (sub specie di impegno, di denuncia, di ricerca di responsabilità) una qualche offerta a chi, per la violenza del potere, è rimasto insepolto ${ }^{6}$. Dando però qui, in Per Isabel, a quella scelta rischiosa un aggiunto significato rituale, vista l'attivazione, tramite il mandala, di quel cerchio di perfezione ed orrore che ricorda, più che il IV capitolo di Notturno indiano, la sua variante beethoveniana costituita dai Treni che vanno a Madras, così come appare nei Piccoli equivoci senza importanza ${ }^{7}$. Già che l'immagine di Shiva danzante sulla quale si giocava almeno in parte l'interpretazione di quella singolare spy story (si ricordi il personaggio che, occultato sotto il nome d'ombra di Peter Schlemihl, si dichiarava convinto, dopo averci pensato "ogni giorno" per anni, di una diversa "interpretazione" della celebre statuetta), portava con sé la forma ciclica del cerchio che il mandala eleva non solo a modalità di costruzione del cosmo ma a modello di individuale e segreto percorso 'iniziatico'. Lungo un cammino teso ad un fine che ha a che fare con il raggiungimento della verità, o quantomeno di una verità provvisoria (vista l'ultima riduzione di ogni percorso al nulla, di ogni ricerca a un cimitero: come avviene in Per Isabel nella mescolanza, nel nono percorso, di presenza ed assenza), e con lo spostamento sempre possibile (il caso anche di Notturno indiano) da un obiettivo all'altro, secondo un procedimento a spirale che è specularmente inverso a quello che ha avvicinato all'oggetto. Significativo a questo proposito il passaggio conclusivo dalle

${ }^{6}$ Il caso di Monteiro Rossi in Sostiene Pereira, del cadavere decapitato nella Testa perduta di Damasceno Monteiro.

${ }^{7}$ Ma per un'analisi in proposito sia consentito il rinvio a Anna Dolfi, Tabucchi, la specularità, il rimorso, Roma, Bulzoni, 2006. 
montagne (della Grecia - come nell'ultimo racconto, Controtempo, del Tempo invecchia in fretta; o della Svizzera, qui, nel ricordo di Hermann Hesse $^{8}$ ), con l'apparizione/ricerca di bizzarri personaggi che vivono fuori del tempo, mescolando strane capacità divinatorie a sapienza orientale.

Quanto al nome di Isabel, inutile dire che si tratta di uno tra più significativi della narrativa tabucchiana: destinataria com'è, in assenza (suo statuto precipuo), la giovane donna (anche tramite la sua 'controfigura', o nom de plume, o doppio speculare, o eteronimo/ortonimo di Magda), della lettera mai spedita che accompagna la sosta al Taj Mahal Inter-Continental Hotel di Bombay nel terzo capitolo di Notturno indiano ${ }^{9}$. La sua tragica fine, mescolata al rimorso e al desiderio di capire, a tentativi di innocentizzazione e a inconsapevoli responsabilità, aveva spinto, in un'estate assolata di deliri, il protagonista di Requiem fino alla prima campata destra del Cemitério dos Prazeres di Lisbona per interrogare un amico morto, scrittore-intellettuale dalla dubbia collocazione politica (a credere almeno a quanto suggerisce un pezzo dell'Angelo nero, Notte, mare o distanza). Ma dall'enigmatico e vagamente demoniaco Tadeus, che al pari di Xavier (l'amico/ alter ego che guida la ricerca di Notturno indiano) si era sempre divertito "a prendere per i fondelli il prossimo", il "timidino", l'interlocutore con "l'anima", il malato di inconscio che percorre le nove stazioni ${ }^{10}$ di Requiem (là dove Tadeus avrebbe dichiarato di non avere che il corpo, e per giunta per

\footnotetext{
${ }^{8}$ Un riferimento a Hesse anche nel VI capitolo di Notturno indiano, nel corso di un colloquio alla Theosophical Society di Madras.

9 "Scrissi a lungo, di getto, con passione, e le raccontai tutto. Le parlai di quei giorni lontani, e del mio viaggio, e di come i sentimenti riaffiorano col tempo. Le dissi anche cose che non avrei mai pensato di dirle" (Notturno indiano, Palermo, Sellerio, 1984, cap. III).

${ }^{10}$ Significativo, in Requiem e in Per Isabel, anche dal punto di vista strutturale, il ritorno insistente sul 9, numero perfetto a cui la simbologia esoterica associa anche capacità di generazione e reincarnazione.
} 
breve durata), non era riuscito a sapere molto, a parte una criptica frase ("è stata tutta colpa dell'herpes zoster", che si scioglierà solo nell'ultima parte del libro) che, mescolata alla ricetta e alla degustazione di un sarrabulho à moda do Douro, rimandava a una dubbia e intrecciata paternità e al senso di colpa ${ }^{11}$. Già che a parlare, a formulare domande, a darsi risposte che non offrivano che un'abbozzata soluzione era solo l'io narrante, che proseguiva il suo viaggio allucinato nei luoghi del ricordo con un appuntamento alla Casa do Alentejo dove una partita di biliardo incredibilmente vinta faceva disegnare sul tavolo strane combinazioni geometriche che, al pari dei mandala, hanno della scommessa (reale e mentale), dello scongiuro, dell'esorcismo, dal momento che erano state capaci (come avverrà anche nel capitolo finale di quest' ultimo libro) di propiziare l'incontro desiderato con Isabel. Anche se di quell'incontro e della donna a lungo cercata il libro 'portoghese' di Tabucchi non ci avrebbe fatto sapere più nulla, visto che l'VIII capitolo di Requiem (romanzo per eccellenza costituito da morceaux choisis, da storie che iniziano divagando fino ad interrompersi a un tratto, rimanendo sospese) metteva in scena soltanto un raccontatore/venditore di storie ed il IX un Convitato/Pessoa che chiudeva circolarmente sul molo una giornata che aveva visto il passaggio dal sole dardeggiante alla notte. Ma è probabile che non potesse essere diversamente, se ogni personaggio, ogni evento (in Requiem come in Per Isabel), non è frutto che della fantasia, dell'allucinazione favorite dalle condizioni necessarie per la creazione e l'ascolto. "La notte è calda, la notte è lunga, la notte è magnifica per ascoltare storie"12, come sostiene l'uomo che in Requiem (memore forse anche del Don Juan Tenorio di Tirso) si siede ai piedi di una statua apprestandosi a declinare generi, tipologie, struttu-

${ }^{11}$ Ma per questo cfr. la sezione "Rimorso e rimosso", Anna Dolfi, Tabucchi, la specularità, il rimorso.

${ }^{12}$ Così l'incipit dell'VIII capitolo di Requiem (Milano, Feltrinelli, 1992). 
re (quelle stesse che il libro di cui parla aveva proposto, e che, in un manieristico tour de force, proporrà il successivo ${ }^{13}$ ).

E una storia (con tutta la capacità affabulativa e perfino divagatoria, ludica, che la parola comporta) è anche quest'ultima, inaspettata, che offre e mescola varianti per il destino di Isabel. Una volta precisato che anche in questo caso - nell'articolazione in cerchi concentrici che dall'evocazione propiziatoria, tramite la ricerca (l'orientamento) e l'identificazione con i luoghi (l'assorbimento), muovono alla reintegrazione e alla ricostituzione dell' immagine — si palesano e occultano ${ }^{14}$ temi ricorrenti, personaggi, letture, ossessioni, incubi, storie sognate (al pari di quelle di Requiem) per effetto di stanchezza, dell'alcool o per eccesso di letteratura in notti di luna piena.

2. Non è un caso che in questo libro che a dispetto di tutto si vuole votivo (il Per non può non ricordare le offerte che sono dovute ai morti) l'evocazione ci riporti a Lisbona, a dare un imprevisto incipit, o a fornire un altro punto di riferimento - d'altronde il punto, il triangolo, il cerchio e il quadrato sono le figure base del mandala - , a quella che finora si poteva pensare come una conclusa trilogia portoghese ${ }^{15}$. Il cammino, la ricerca (ce ne avevano resi edotti Notturno indiano e Requiem, che con il loro andamento circolare costituiscono gli altri due lati del triangolo ora chiuso da Per Isabel ${ }^{16}$ ) inizia con una sosta in un piccolo caffe dove un liquore, benché non

${ }^{13}$ In effetti Per Isabel si potrebbe anche leggere come raccolta delle diverse declinazioni di una storia a protagonisti fissi ma a personaggi variabili (attraverso un allargamento della maniera di Queneau).

${ }^{14}$ Come la nota d'apertura al libro dichiara, mescolando secondo l'uso il rivelare e il nascondere.

${ }^{15}$ L'espressione appartiene alla bella prefazione di Carlo Feltrinelli a un recente assemblage tabucchiano (Romanzi. Requiem. Sostiene Pereira. La testa perduta di Damasceno Monteiro, Milano, Feltrinelli, "Le comete", 2012).

${ }^{16}$ Lo prova anche la singolare ricorrenza nei tre libri di uno stesso sintagma, singolarmente significativo (ma per questo si veda Anna Dolfi, "Le storie di un libro. Postfazione", Antonio Tabucchi, Di tutto resta un poco, p. 276) o per citazione diretta 
d'annata (come invece in Requiem), accompagna una possibile scommessa a biliardo. Anche la posta in gioco è sempre la stessa, ma questa volta il personaggio alla ricerca di Isabel è un "angelo della notte" (Nightingale contro Rouxignol, insomma, per usare i nomi/volti speculari di Notturno indiano) e si chiama Tadeus. Un Tadeus lunare, che si colloca ironicamente (o veridicamente, vista l'associazione invalsa di Sirio con il caldo e con possibili sciagure) nella costellazione del Cane Maggiore, pronto a muoversi (nel breve congedo, ma da dove? dalla morte forse, che lo aveva immobilizzato al Cemitério dos Prazeres?), come già il protagonista di Requiem, in uno spazio di specchi, di cristalli, di delirio, di sogni. Anche qui - anche se non ne avevamo mai saputo tanto: se ne dichiara perfino il nome, Isabel Queiroz do Monte - la figura femminile apparirà di sfuggita, "perché i personaggi rovinano il mistero" (come recita il testo) ed è importante lasciare aperte le possibilità, come avviene per le finestre vuote nei quadri di Isabel, per le figure appena abbozzate nei racconti e nei romanzi non finiti (che poi sono la regola) di Tabucchi... Intanto ranocchie alla provenzale (le grenouilles ricorrono spesso nei racconti o nel romanzo per lettere ${ }^{17}$ ) accompagnano una delle tante possibili suggestioni di morte, si potrebbe dire uno degli ultimi inviti: quello all'impiccagione, che era stato anche dell'Antigone sofoclea, condannata ad essere sepolta viva. Ma è normale che delle donne angelicate non si sappia troppo. Per altro, non c'è nessun dubbio che, nonostante l'anticonformismo, Isabel appartenga alla loro schiera. Basta qualche tratto per inscriverla nel mito montaliano di Clizia ${ }^{18}$ : il cappello

("di tutto resta un poco"), o per evocazione del suo autore (Drummond de Andrade, citato nella "Nota" d'apertura a Requiem).

${ }^{17}$ Si sta facendo sempre più tardi, Milano, Feltrinelli, 2001, all'interno del quale si trova un riferimento a un romanzo che potrebbe essere Per Isabel.

${ }^{18}$ Per alcune presenze montaliane nella narrativa di Tabucchi si veda il saggio di una mia allieva, Marica Romolini ("I notturni della coscienza: il montalismo degli angeli neri”, I'notturni' di Antonio Tabucchi, a cura di Anna Dolfi, Roma, Bulzoni, 2008, pp. 119-129). 
di paglia di Firenze che porta con sé (e che riapparirà fino alla copertina di Si sta facendo sempre più tardi), il capretto al guinzaglio (memore forse degli sciacalli del mottetto delle Occasioni, "La speranza di pure rivederti"), lo sfondo sonoro fatto dall'ibridazione di musiche nobili e popolari, costante nella Bufera, assieme all'orfanità diffusa che segna la fine dell'infanzia (altro sintagma montaliano, assieme al ricordo di una "casa sul mare", già evocata in Requiem a proposito di Isabel), e alla sciarpa (che è sempre al collo del visiting angel), al gesto con la mano che accompagna l'addio (su cui si era chiuso anche Requiem), prima della discesa nel buio (così nella poesia eponima della Bufera).

Studentena in Lettere moderne, quasi come il 'filosofo' in erba Monteiro Rossi di Sostiene Pereira, anche Isabel mescolerà nell'elogio funebre per García Lorca letteratura e politica e - almeno nella versione fornita dalla vecchia governante (ma una frau fedele sarà l'unica vera compagnia anche della calda estate di Tristano ${ }^{19}$ ) — scomparirà con un pacco di manifestini; mentre, nonostante la ben più giovane età, fados castiços e candele accese ne fanno un personaggio misterioso e intrigante come la Maria do Carmo del Gioco del rovescio. D'altronde il ragazzo straniero, forse spagnolo, a cui si era accompagnata secondo una prima testimonianza, assieme allo scrittore polacco in cui è facile riconoscere Tadeus (quest'ultimo cambierà poi nel corso del libro il nome di Wacław in quello del poeta romantico Juliusz Słowacki) fa parte, quanto a predilezioni culturali (Vittorini...), della famiglia dei Monteiro e dei Firmino ${ }^{20}$, mentre il cane giallo pare anticipare

${ }^{19}$ Tristano muore, Milano, Feltrinelli, 2004. Ma per una lettura di quel libro e delle ibridazioni testuali con la letteratura italiana e europea (di cui qualche traccia anche in Per Isabel) cfr. Anna Dolfi, “'Le temps pressé’ e 'le vouloir écrire' di Tristano”, Gli oggetti e il tempo della saudade. Le storie inafferrabili di Antonio Tabucchi, Firenze, Le Lettere, 2010, pp. 73-87.

${ }^{20}$ Il giornalista cosmopolita che ne La Teste perdute di Damasceno Monteiro studia Vittorini e sogna di scrivere un saggio sulla sua influenza sul romanzo portoghese del dopoguerra. 
quello, alla Goya, di Tristano muore ${ }^{21}$. Certo è che la ricostruzione della morte di Isabel (un suicidio per depressione dopo un aborto procurato), così com'era stata adombrata in Requiem, è qui presentata e smontata come una delle tante soluzioni possibili, ad alimentare il rimorso, a crescere il mito dell'eroismo, a nascondere un delitto politico, a nutrire la speranza della salvezza. Anche se a ritornare saranno, e fino all'ultimo, soprattutto immagini di morte (per impiccagione, per ingestione di pezzi di vetro, per assunzione di tranquillanti, per tempo ormai inevitabilmente passato) e di clausura, come era avvenuto nel caso di Antigone, costretta u una grotta a cui potrebbero forse vagamente alludere la Boca do Inferno vicina a Cascais, alla cui visita invita, dopo delle esequie dilazionate (ovvero una rituale celebrazione in assenza di corpo), un cameriere della baia ${ }^{22}$, o la Grotta di Camões di Macao, ove su ispirazione delle stelle Tadeus tenta il sesto circolo, quello della comunicazione. Ma si tratterà di una comunicazione indiretta, visto che per sapere di Isabel si dovrà ascoltare la voce di Magda, acconsentire insomma (a voler continuare con la provocazione montaliana, dagli Xenia questa volta o dalle prose della Farfalla di Dinard ${ }^{3}$ ) che Clizia lasci spazio a Mosca e al suo infallibile "radar di pipistrello" ${ }^{24}$. E non si tratterà questa volta di un gioco nominalistico, ma di una sostituzione vera (analoga a quella che inversamente aveva aperto, per poi annullarle alla fine,

${ }^{21}$ Su cui si veda Nives Trentini, "Dalle Tentazioni di Bosch al 'Cane' di Goya. La tonalità notturna di Tristano muore”, I 'notturni' di Antonio Tabucchi, pp. 315-334.

${ }^{22}$ Un altro barman si intratterrà invece nel terzo circolo con l'io narrante in un rapporto analogo, per conoscenza e complicità, a quello stabilito da Pereira al Café Orchídea.

${ }^{23}$ Proprio per il ruolo svolto dal pipistrello in quelle prose cfr. lo splendido saggio di Adelia Noferi, " 'Cripte, buche e nascondigli' in Montale", Nevrosi e follia nella letteratura moderna. Atti di seminario. Trento, maggio 1992, a cura di Anna Dolfi, Roma, Bulzoni, 1993, pp. 478-491 (poi in Adelia Noferi, Soggetto e oggetto nel testo poetico. Studi sulla relazione oggettuale, Roma, Bulzoni, 1997).

24 "Non ho mai capito se io fossi" (dagli Xenia di Satura). 
le immagini distinte degli amici in Notturno indiano), necessaria a fornire le ultime indicazioni per il viaggio spingendo ai limiti della temporalità, fino al settimo circolo. Quello più vicino ai giochi di parole, alle inquietanti creazioni della letteratura che hanno vita non avendola, e che esistono pur non avendo storia. Glasperlenspiel, "gioco delle perle di vetro" collocate sulla liminarità tra reale e fantastico, astrazione e fisica concretezza. La conoscenza raggiunta grazie a una raffinata composizione di pezzi, a uno spostamento di tessere che consente di costruire il racconto a partire da una serie di varianti che vanno ogni volta in direzioni diverse (con una tendenza al divertissement che ha a che fare col gioco di prestigio), portando alla dilatazione là dove tutto può di nuovo ritrovarsi e convergere, le religioni come i piatti tipici (e con una gamma di frequenza che include un arco narrativo che va dal Ganesh di Notturno indiano alle ricette di Requiem, agli incontri e ai personaggi — Xavier in testa — che in quei libri si susseguono senza alcuna apparente soluzione di continuità). Per non parlare degli strani e inquietanti segni/segnali di riconoscimento (ancora montaliani) che uniscono il mondo dei vivi e quello dei morti, il nulla e il tutto, il presente e il passato in un'assolutoria abolizione del tempo. Nella pace cimiteriale offerta, infine, nel nono circolo, da un ritorno che è realizzazione, e da una realizzazione che è di nuovo possibilità di apparire. Come nella musica beethoveniana evocata da Les adieux ${ }^{25}$, dove l'abbandono e il rimpianto dell'incipit lasciano spazio ai movimenti successivi: l'andante dell'assenza, il vivace gioioso del ritorno. Anche se poi, finita la melodia, l'apparizione si rivelerà nella sua natura onirica e allusiva (ma era già avvenuto in Requiem, forse anche in Notturno indiano), e a rimanere non sarà che una sinopia (giacché cosa conserva, la fotografia, di quel che fu reale? non è forse ogni

\footnotetext{
${ }^{25}$ Nelle ultime pagine di Requiem dopo la fisarmonica, i fados, Chopin..., c'era stata la musica per pianoforte di Liszt.
} 
andare un percorso tra ombre?), insieme alla sciarpa bianca e all'addio. Abbandonata la "speranza di pure rivederti" (come aveva sillabato Montale), nonostante che si sia sgombrato lo "schermo di immagini" che fino ad allora aveva impedito la vista, consentendo comunque che nella mente, di volti, a campeggiare ne resti uno solo. Ma, dopo il congedo, proiettato in alto, tra le stelle, sullo sfondo della volta celeste, quasi in offerta compensatoria per la ricerca e l'attesa.

3. Nelle sue Note all'Antigone (è da quelle poche pagine - per l'esattezza dalla loro seconda sezione - che vengono l'esergo e le parole di Hölderlin citate nella Giustificazione in forma di nota con i quali si apre Per Isabel) il grande poeta tedesco si soffermava sul rapporto che nella tragedia sofoclea esiste tra l'avvio e la conclusione, sul ritmo delle scene, sulla dinamica mutevole delle parti, sull'inesorabile direzionalità narrativa di quel testo verso il suo compimento, ma anche su quelle che potremmo liberamente chiamare le improvvise accelerazioni dello spleen e sulle forze che diversamente conducono la sfera solare e quella notturna, il mondo dei vivi e quello dei morti. Fino a spingere un lettore/scrittore moderno come Tabucchi (partito forse anche dal viaggio di Pedro Páramo ${ }^{26}$ ) a un'ennesima discesa nell'Ade dopo quella di Requiem. Per delineare una mitologia/mitografia nella quale, con le modalità della simmetria e dell'eccesso, tutto si confonde nel gioco del rovescio ("i morti hanno altro costume"), e le voci delle figure profetiche (il Tiresia di Sofocle, l'eremita di Hölderlin...), si intrecciano ad altre, depistanti e diversamente persecutorie. D'altronde, laddove Zeus o la

\footnotetext{
${ }^{26}$ Significativo che a Tabucchi sia capitato anche negli ultimi tempi, in un appunto inedito (citato da Maria José de Lancastre nella sua "Premessa" a Fernando Pessoa, Poesie di Fernando Pessoa, a cura di Antonio Tabucchi e Maria José de Lancastre, Milano, Adelphi, 2013), di parlare di Juan Rulfo come di uno scrittore assoluto (“E evidente che in letteratura il genio si possa rivelare anche in un solo libro (penso al Pedro Páramo di Juan Rulfo, a L'étranger di Camus)”).
} 
notte hanno stanza, è consentito sospendere l'incredulità, inseguire i fantasmi, mescolare il "fingere" e il "sentire" 27 , sognando sotto la volta celeste, come un tempo in compagnia di Pessoa, guidati da una traduzione ottocentesca dei tragici greci $^{28}$. La coscienza in ogni caso, tra bene e male, theos e antitheos (chi ha detto infatti — ove si segua alla lettera la replica di Antigone - che sia giusto riservare un trattamento diverso a innocenti e a colpevoli?), non potrà salvare o perdere che Isabel. Per questo il viaggio fatto per "nostalgia dei prototipi" (come avrebbe detto Bernardo Soares), che porta il pellegrino d'amore disceso nel mondo dei morti a rivedere le stelle, potrà essere affidato a Tadeus. La sua anima, buona o cattiva che sia (giacché non è sicuro che il regno misterioso nel quale conduce la poesia obbedisca alle stesse regole di quello umano nel quale crediamo di vivere), dovrà muoversi in un mondo alternativo (montalianamente parallelo), là dove è consentita ogni metamorfosi, di situazioni, di personaggi, di figure, perfino di metafisica ${ }^{29}$.

${ }^{27}$ Così nel IX capitolo di Requiem.

${ }^{28}$ Nello specifico di Sofocle e della sua Antigone (che — varrà ricordarlo, per citare solo altri autori cari a Tabucchi — sarebbe giunta fino a Brecht e a Maria Zambrano).

${ }^{29}$ Di nuovo pessoana: si pensi al "tutto è niente" che dal quarto circolo di Per Isabel riconduce al Libro dell'inquietudine di Bernardo Soares: "Tutto è noi e noi siamo tutto; ma a che serve questo, se tutto è niente?"; "tutto è niente" (dalle citazioni 12 (67) e 65 (434) da Fernando Pessoa, Il libro dell'inquietudine di Bernardo Soares, rispettivamente pp. 35 e 88 ). 